\title{
GC-MS Analysis and Phytochemical Screening of Indigofera tinctoria (Linn.) Leaf Extract Characterizing its Medicinal Use
}

\author{
Research Article
}

\section{Mishra DN1, Gomare KS2*, Sheelwant SV²}

\author{
1. PAH Solapur University, Kegaon, Solapur, (MS), India \\ 2. Biotechnology Research Centre, COCSIT, Latur, (MS), India
}

\begin{abstract}
Indigofera tinctoria (Linn.), a perennial shrub belongs to the family Fabaceae (Leguminosae) is well known in Indian system of medicines for its traditional uses. The present study was carried to find out the possible bioactive components in the leaves of this plant in ethanol extract by Gas Chromatography-Mass Spectrometry (GC-MS) analysis and the therapeutic uses of each compound as per PASS and other database. The mass spectra of the extract found compounds that were matched with the National Institute of Standards and Technology (NIST version 5.7.1) library database. The GC-MS study observed different peaks as per retention time and then those peaks were analysed determining presence of 26 phytochemical compounds. The qualitative phytochemical screening done by routine laboratory methods also indicated presence of these bioactive compound groups like alkaloids, flavonoids, tannins and phenols, saponins, glycosides and terpenoids. These 26 compounds are understood to have different therapeutic activities as per in-silico databases, e.g. 2-Acetylamino-3-hydroxy-propionoic acid (glutamic acid) - used as Anti-diarrheal, Antiviral, Antipyretic, Protein synthesis inhibitor, Antidiabetic, Non-steroidal, Anti-inflammatory agent, Antipsoriatic, Antioxidant, Antifungal, Antineoplastic (sarcoma), Antiparasitic, Antibiotic Aminoglycosidelike, Antiseborrheic, Anticataract, Antithyroid, Anticarcinogenic, Antileprosy, Hair growth stimulant, Lipoprotein lipase inhibitor, Dermatologic, Testosterone, Antituberculosic, Antirickettsial, Antianemic, etc. activities. Hence, Indigofera tinctoria is an excellent source of phytocompounds, which help to heal various diseases and health complications in human beings. The use of this plant in traditional methods for the treatment of such diseases appears scientifically relevant as per the therapeutic activity data of its bioactive compounds.
\end{abstract}

Key Words: Indigofera tinctoria, GC-MS, Therapeutic activities, NIST library, Medicinal plants.

\section{Introduction}

Indigo plant is a common name given to any of a genus of perennial herbs/ shrubs that reflect indigo colour during post flowering. The group has about 700 species, most native to tropical regions. Various Asian species contain the glycoside indican, which can be oxidized to produce the dyestuff indigo. Indigofera tinctoria (Linn.) is one such prominent plant that was cultivated in India due to its high trading value and was known as blue gold and true indigo. Previously, various parts of this plant were reported in use for treatment of different types of diseases, especially of Indigo powder (The leaves were soaked in water and fermented to convert the glycoside indican present in the plant to the blue dye indigotin) being used in Indian traditional medicine for the treatment of removing worms in teeth and gums, sores on skin, urinary problems, mouth ulcers, hair rejuvenation, skin diseases and dog bite, etc.

\section{* Corresponding Author:}

\section{Komal S Gomare}

Research Scholar, Department of Biotechnology,

College of Computer Science and Information

Technology, Ambajogai Road, Latur - 413531,

Maharashtra (MS), India

Email Id: komalgomare2007@rediffmail.com
(1-3). Indigofera tinctoria has been identified from the descriptions of formulations to deal with different health disorders from Charaka Samhita, Sushruta Samhita, Ashtanga Hridayam (Brihattrayi) and Madhava Chikitsa like Sanskrit medical texts. The plant is described for having Rasa (=Taste): Katu and Tikta; Gunas (=Quality): Sara; Virya (=Active constituent): Ushna (driving force behind the therapeutic activity of the drug, which signifies potency of a medicinal plant/ drug); Vipaka (=Final outcome): Katu and Karma (=action): Kaphahara, Keshya, Vatahara.

The reason of medicinal use of many plants is assigned to the active parts of each plant, where some biologically active compounds are stored by the plants [biological activities like- antimicrobial, antioxidant, anti-inflammatory, antiseborrheic, antipsoriatic and antiacne, etc.] $(3,4)$. Eventually, these biologically active compounds / phytochemicals are gaining importance for the understanding of the therapeutic applications. People are returning to natural products/ practices to address many of their chronic health disorders. Such products; some containing herbal parts and some other containing nutritional agents in the market claim to have positive effects.

GC-MS analysis is a strong scientific process to identify and characterize presence of phytocompounds in the plant parts. At the same time, sophisticated in- 
silico bioinformatics software tools, such as PASS (Prediction of Activity Spectra for Substances) explore the hidden pharmacological potential of the phytoconstituents from the database. The future drug development is mostly going to be monitored from the redefined lead compounds identified from such integrated studies $(5,6)$. Literature survey revealed that till date, no PASS prediction has been reported on phytocompounds (GC-MS analysis) from Indigofera tinctoria leaves with therapeutic activities. Therefore, present study was focused on bioactive phytocompounds from ethanol extract of the plant leaves to open up the future possibilities.

\section{Materials and Methods}

Collection of plant material: Indigofera tinctoria plant (locally known as Neel or indigo) was collected from the Dhanvantari Udyaan, Mahatma Phule Krishi Vidyapeeth, Rahuri, Maharashtra, India.

Preparation of extract using Soxhlet apparatus: $20 \mathrm{~g}$ of dry leaves powder was placed in the body of soxhlet extractor. $250 \mathrm{ml}$ of solvent-ethanol placed into round bottom flask of the soxhlet apparatus. The process of filling and emptying of the extractor was repeated until the drug is exhausted. Then soxhlet solvent containing extract was poured into large petridish and allowed to evaporate. The final crude extract was stored in a refrigerator at $4{ }^{\circ} \mathrm{C}$ for further use during phytochemical analysis.

Phytochemical analysis: The preliminary qualitative phytochemical screening of ethanol extract was carried out according to the standard laboratory procedure (7).

GC-MS analysis: GC-MS analysis and characterization of ethanol leaves extracts of Indigofera was done with EI- MS Spectrum scanned at $70 \mathrm{eV}$ at SAIF, IIT Bombay, India. The sample was run for 35 minutes. NIST version (5.7.1)(8), Pubchem $(9,10)$, PASS (11), IMPPAT (12), NCIt (13), HMBD (14), ChEBI (15), MeSH (16) and Drugbank (17) chemical/ pharmaceutical databases were used to identify and characterize the spectra revealed molecules.

\section{Results}

The qualitative phytochemical analysis of Indigofera ethanolic extract of leaves was performed by using different tests and the results are presented in Table 1. The presence of alkaloids, flavonoids, tannins and phenols, saponins, glycosides and terpenoids were found and steroids, anthraquinones were not found present when tested for the extracts.

\section{Table 1: Qualitative Phytochemical analysis of leaves extracts of Indigofera tinctoria}

\begin{tabular}{|r|l|l|l|c|}
\hline Sr. No. & \multicolumn{1}{|c|}{ Constituents } & \multicolumn{1}{|c|}{ Test } & Expected Results & Leaves \\
\hline 1 & Alkaloids & Wanger's test & Brown ppt. & + \\
\hline 2 & Flavonoids & Ammonia $/ \mathrm{H}_{2} \mathrm{SO}_{4}$ test & Yellow colour appears \& disappears later & + \\
\hline 3 & Tannins \& Phenols & Lead acetate & Yellow ppt. & + \\
\hline 4 & Saponins & Frothing test & Foamy layer & - \\
\hline 5 & Steroids & Salkowski test & Red color in chloroform layer & - \\
\hline & & Libermann Burchard Test & Brown ring at junction & + \\
\hline 6 & Glycosides & Salkowski test & Red color in chloroform layer & + \\
\hline 8 & Anthraqui-nones & Chloroform test & Interface reddish brown & - \\
\hline
\end{tabular}

The GC-MS spectrum of ethanolic extract of Indigofera leaves is depicted in Figure 1 and individual mass spectrum of phytocompound is illustrated in Figure 2. In GC-MS analysis total 26 phytocompounds were identified. The identification of phytocompounds is based on molecular formula and retention times (RT) being compared to the database of NIST and the further collected information on therapeutic activities of each compound was done from Pubchem, PASS, IMPPAT, Drugbank, NCIt, HMBD, ChEBI and MeSH. Findings are presented in Table 2. It has been largely observed and driven that many structurally related compounds (i.e. mostly Maximum Common Substructure or MCS) display similar bioactivity and physicochemical properties (18).

Figure 1: GC-MS spectrum of ethanolic extract of Indigofera tinctoria

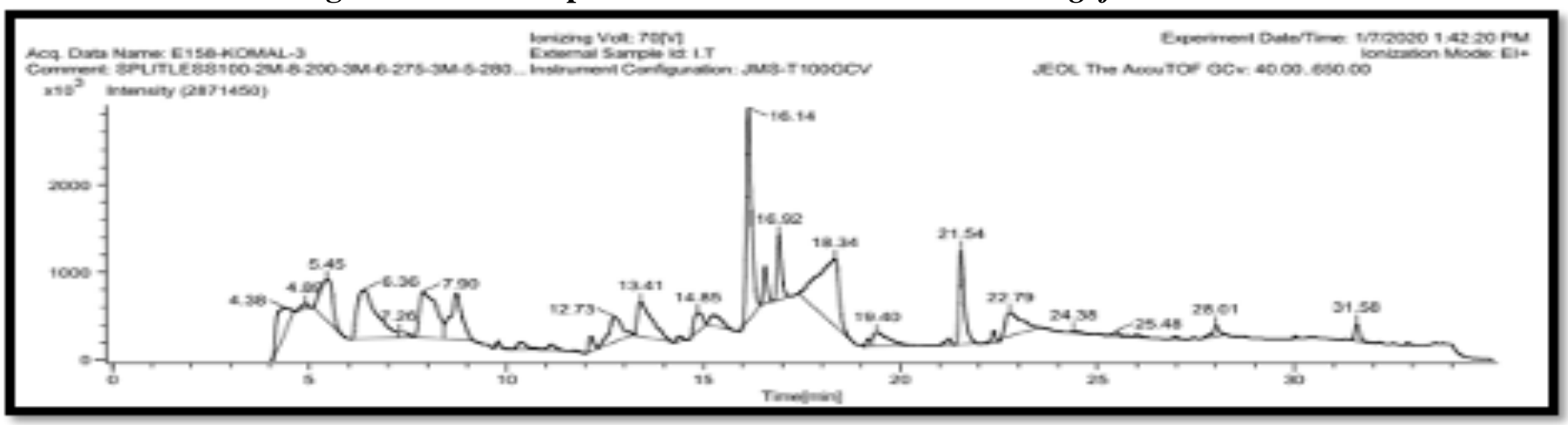


Figure 2: Mass spectrum of each identified phytocompound

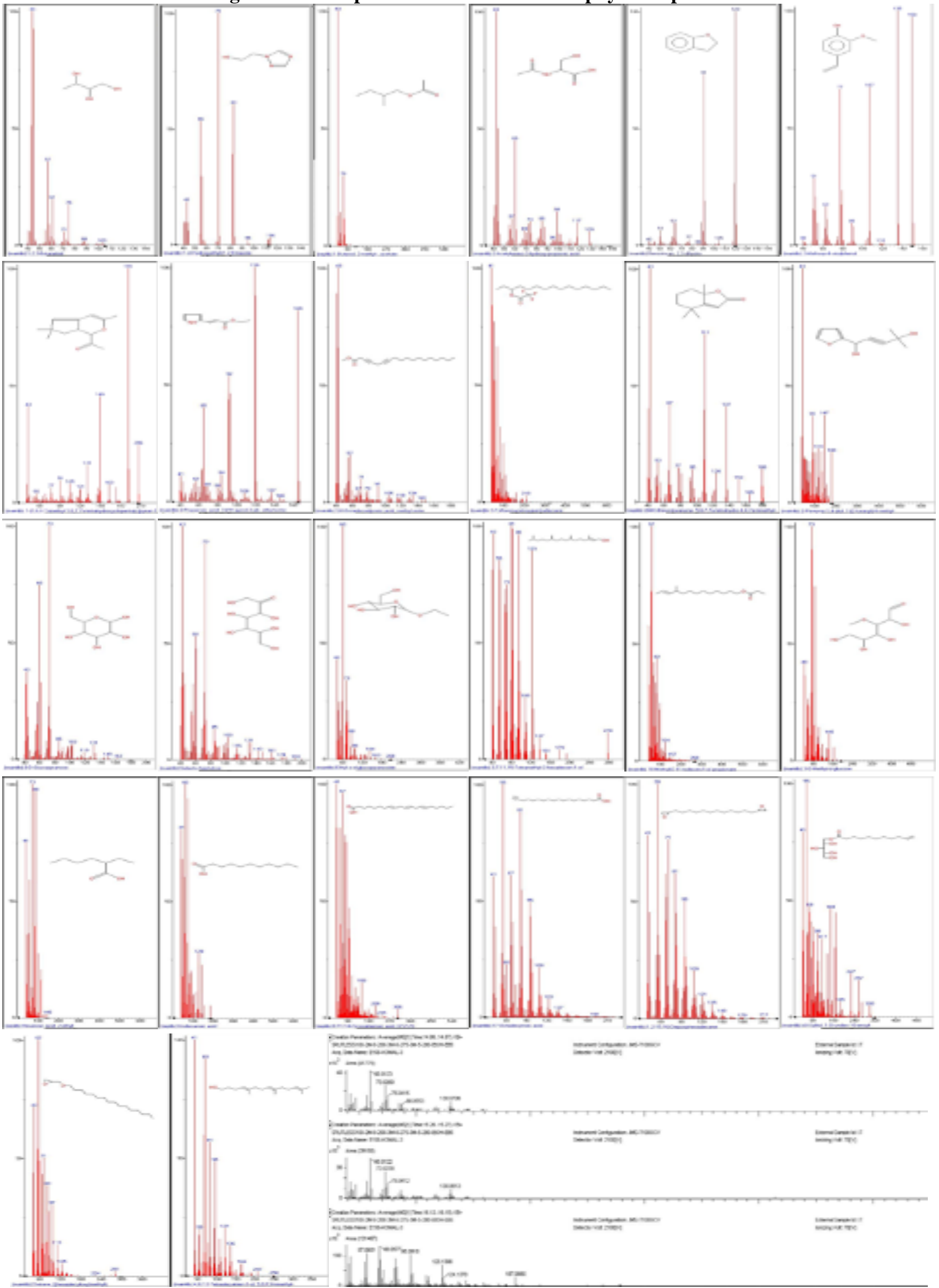


Table 2: Phytocompounds identified and the therapeutic activities of each compound

\begin{tabular}{|c|c|c|c|c|}
\hline $\begin{array}{l}\text { Sr. } \\
\text { No. }\end{array}$ & $\begin{array}{l}\text { Name of phyto- } \\
\text { compounds }\end{array}$ & $\begin{array}{l}\text { RT } \\
(\min )\end{array}$ & $\begin{array}{l}\text { Molecular } \\
\text { Formula }\end{array}$ & $\begin{array}{l}\text { Therapeutic activity } \\
\text { (Pubchem, PASS* and IMPPAT**)(NCIt, HMBD, } \\
\text { ChEBI, MeSH, Drugbank) }\end{array}$ \\
\hline 1 & 1,2,3 Butanetriol & 4.89 & $\mathrm{C}_{4} \mathrm{H}_{10} \mathrm{O}_{3}$ & $\begin{array}{l}\text { Phobic disorders treatment, Antiseborrheic, } \\
\text { Phospholipase inhibitor, Testosterone 17beta- } \\
\text { dehydrogenase (NADP+) inhibitor, Hair growth } \\
\text { stimulant, Antipsoriatic, Antidiabetic, Non-steroidal } \\
\text { antiinflammatory agent, Antiviral (Hepatitis B), } \\
\text { Antiallergic, Antieczematic atopic, Antibacterial, } \\
\text { Antibiotic Anthracycline-like, Antifungal,Antibiotic } \\
\text { Aminoglycoside-like, Antiparkinsonian, Antineoplastic } \\
\text { (breast cancer), Antineoplastic antibiotic, } \\
\text { Antiadrenergic }\end{array}$ \\
\hline 2 & $\begin{array}{l}\text { 1-(2- } \\
\text { Hydroxyethyl-1,2,4- } \\
\text { triazole }\end{array}$ & 5.45 & $\mathrm{C}_{4} \mathrm{H}_{7} \mathrm{~N}_{3} \mathrm{O}$ & $\begin{array}{l}\text { Antiviral (Adenovirus) \& (Influenza A), Kinase } \\
\text { inhibitor, Antileprosy,Antineurotic, Antibacterial, } \\
\text { ophthalmic, Antiparkinsonian, Antirickettsial, } \\
\text { Antiinflammatory (ophthalmic), Antidiarrheal, } \\
\text { Antiseborrheic, Antialcoholic, Antiparasitic, } \\
\text { Antidiabetic, Antianemic, Antineoplastic (lymphocytic } \\
\text { leukemia), Antibiotic Trimethoprim-like, Hair growth } \\
\text { stimulant, Testosterone 17beta-dehydrogenase } \\
\text { (NADP+) inhibitor, Lipoprotein lipase inhibitor }\end{array}$ \\
\hline 3 & $\begin{array}{l}\text { 1-Butanol, 2-methyl, } \\
\text { acetate }\end{array}$ & 6.37 & $\mathrm{C}_{7} \mathrm{H}_{14} \mathrm{O}_{2}$ & $\begin{array}{l}\text { Antieczematic, Antiseborrheic, Antiviral, Hair growth } \\
\text { stimulant, Antiulcerative, Antiinflammatory, } \\
\text { Antipyretic, Antiparkinsonian, Antiparasitic, Yeast } \\
\text { ribonuclease inhibitor, Dermatologic, Anticataract, } \\
\text { Antipsoriatic, Antileprosy, Antituberculosic, } \\
\text { Antialcoholic, Antifungal, Antirickettsial, Antiallergic } \\
\text { Non-steroidal antiinflammatory agent, Antiinfertility } \\
\text { (female), Anticarcinogenic, Antidiabetic, Antithyroid, } \\
\text { Antidepressant, Imipramin-like, Antibacterial } \\
\text { (ophthalmic), Antineoplastic (endocrine cancer), } \\
\text { Antioxidant, Antiischemic, Antianemic, Antifungal, } \\
\text { Antidiarrheal, Lipoprotein lipase inhibitor, } \\
\text { Dermatologic, Testosterone 17beta-dehydrogenase } \\
\text { (NADP+) inhibitor }\end{array}$ \\
\hline 4 & $\begin{array}{l}\text { 2-Acetylamino-3- } \\
\text { hydroxy-propionoic } \\
\text { acid (glutamic acid) }\end{array}$ & 7.37 & $\mathrm{C}_{5} \mathrm{H}_{9} \mathrm{NO}_{4}$ & $\begin{array}{l}\text { Antidiarrheal, Antineoplastic (lymphocytic leukemia), } \\
\text { Antiviral, Antipyretic, Protein synthesis inhibitor, } \\
\text { Antidiabetic, Non-steroidal antiinflammatory agent, } \\
\text { Antipsoriatic, Antioxidant, Antifungal, Antiparasitic, } \\
\text { Antibiotic Aminoglycoside-like, Antiseborrheic, } \\
\text { Antieczematic, Antialcoholic, Antiinflammatory } \\
\text { (intestinal), Antiulcerative, Anticataract, non-allergic, } \\
\text { Antithyroid, Anticarcinogenic, Antibiotic Glycopeptide- } \\
\text { lik e, A n t i l e p r o y, H a i r g r o w th } \\
\text { stimulant,Antituberculosic, Antidiabetic, Antirickettsial, } \\
\text { Antianemic, Lipoprotein lipase inhibitor, Dermatologic } \\
\text { Testosterone 17beta-dehydrogenase (NADP+) inhibitor }\end{array}$ \\
\hline 5 & $\begin{array}{l}\text { Benzofuran, 2,3- } \\
\text { dihydro- }\end{array}$ & 7.90 & $\mathrm{C}_{8} \mathrm{H}_{8} \mathrm{O}$ & $\begin{array}{l}\text { Antiinflammatory, Antiseborrheic, Antiviral, } \\
\text { Antipsoriatic, Anticataract, Antiseptic, Antifungal, } \\
\text { Antirickettsial, Antiparkinsonian, Antipyretic, } \\
\text { Antibacterial, Antileprosy, Antieczematic, Antidiabetic, } \\
\text { Antimycobacterial, Anticarcinogenic, Antidepressant, } \\
\text { Imipramin-like, Antialcoholic, Antibiotic Macrolide- } \\
\text { like, Antianemic }\end{array}$ \\
\hline
\end{tabular}




\section{6}

2-Methoxy-4-

\begin{tabular}{|l|l|}
\hline vinylphenol \\
\\
\hline 7 & $1-(3,6,6-$
\end{tabular}

\subsection{3}

$\mathrm{C}_{9} \mathrm{H}_{10} \mathrm{O}_{2}$
7
1-(3,6,6-
Trimethyl-1,6,7,7a-
tetrahydrocyclopenta

(c) pyran-1-
yl)ethanone

\begin{tabular}{|l|l|}
\hline $\begin{array}{l}\text { (c) pyran-1- } \\
\text { yl)ethanone }\end{array}$ \\
\hline $8 \quad \begin{array}{l}\text { 2-propenoic acid,3- } \\
\text { (1H-pyrrol-2yl)- } \\
\text { ethyl ester }\end{array}$ \\
\hline
\end{tabular}

$9 \quad$ 3,6-Octadecadiynoic 11

\begin{tabular}{|l|l|}
\hline acid, methyl ester \\
\hline
\end{tabular}

$9.79 \quad \mathrm{C}_{13} \mathrm{H}_{18} \mathrm{O}_{2}$

$10.37 \mathrm{C}_{9} \mathrm{H}_{11} \mathrm{NO}_{2}$

$11.13 \quad \mathrm{C}_{19} \mathrm{H}_{30} \mathrm{O}_{2}$

\begin{tabular}{|l|l|}
\hline 10 & $3-$ \\
& $\begin{array}{l}\text { Trifluoroacetoxypen } \\
\text { tadecane }\end{array}$
\end{tabular}

$11.70 \quad \mathrm{C}_{17} \mathrm{H}_{31} \mathrm{~F}_{3} \mathrm{O}_{2}$

$12.15 \mathrm{C}_{11} \mathrm{H}_{16} \mathrm{O}_{2}$
Antiseborrheic, Testosterone 17beta-dehydrogenase (NADP+) inhibitor, Antileukemic, Antiischemic, Antiinflammatory, Alopecia treatment, Antiviral, Antiinfertility, Antipyretic, Lipoprotein lipase inhibitor, Phospholipase inhibitor, Antifungal, Antiseptic, Antiacne, Antipsoriatic, Antiparkinsonian, Anticarcinogenic, Antileprosy, Antirickettsial, Antibacterial, Antithyroid, Antidiabetic, Hair growth stimulant, Antiosteoporotic, Antidepressant (Imipraminlike), Antieczematic, Antioxidant, AntimitoticPodophyllotoxin-like, Antibiotic Anthracycline-like

Antiinflammatory, Phobic disorders treatment, Testosterone 17beta-dehydrogenase (NADP+) inhibitor, Antipyretic, Antieczematic, Antiacne, Antiseborrheic, Lipoprotein lipase inhibitor, Antipsoriatic, Antiviral, Antiparkinsonian, Anticarcinogenic, Anticataract, Hair growth stimulant, Antifungal, Antithyroid, Antioxidant, Antibacterial, Antituberculosic, Antidiabetic, Antibiotic Glycopeptide-like, Antirickettsial

Antiseborrheic, Testosterone 17beta-dehydrogenase (NADP+) inhibitor, Lipoprotein lipase inhibitor, Antiviral, Antipyretic, Antiinflammatory, Hair growth stimulant Antituberculosic, Antiseptic, Antimycobacterial, Antiparkinsonian, Antileprosy, Antipsoriatic, Antirickettsial, Antialcoholic, Phospholipase inhibitor, Antithyroid, Antiparkinsonian, Antifungal, Antibacterial-ophthalmic, Antiinfertility, Anticataract, Antidiabetic

JAK2 expression inhibitor, Antiseborrheic, Testosterone 17beta-dehydrogenase inhibitor, Lipoprotein lipase inhibitor, Phospholipase inhibitor, Hair growth stimulant, Antiosteoporotic, Antiviral, Antiacne, Antiinfertility-female, Antiinflammatory, Antieczematic, Antibacterial, Anticarcinogenic, Antialcoholic, Antineoplastic (pancreatic cancer), Antiparkinsonian-rigidity relieving, Antifungal, Antioxidant, Antidiabetic, Antirickettsial

Testosterone 17beta-dehydrogenase (NADP+) inhibitor, Antiviral, Antiinflammatory, Lipoprotein lipase inhibitor, Antiseborrheic, Antipsoriatic, Phospholipase inhibitor, Antibacterial, Antipyretic, Antidiabetic, Anticarcinogenic, Antithyroid, Antirickettsial, Hair growth stimulant, Antileprosy, Antiinflammatory, Antieczematic, Antiparkinsonian

Antiseborrheic, Antieczematic, Testosterone 17betadehydrogenase (NADP+) inhibitor, Phobic disorders treatment, JAK2 expression inhibitor, Antipsoriatic, Lipoprotein lipase inhibitor, Phospholipase A1 inhibitor, Wound healing agent, Antiasthmatic, Antioxidant, Antineoplastic (small cell lung cancer), Antidiabetic, Antidiarrheal, Antidepressant-Imipramin-like 


\begin{tabular}{|c|c|c|c|c|}
\hline 12 & $\begin{array}{l}\text { 2-Pentyne-1,4,- } \\
\text { diol,1-(2-furanyl)-4- } \\
\text { methyl- }\end{array}$ & 12.73 & $\mathrm{C}_{10} \mathrm{H}_{12} \mathrm{O}_{3}$ & $\begin{array}{l}\text { Phobic disorders treatment, Antiseborrheic, Lipoprotein } \\
\text { lipase inhibitor, Antieczematic, Antimutagenic, } \\
\text { Testosterone 17beta-dehydrogenase (NADP+) inhibitor, } \\
\text { Prostaglandin-E2 9-reductase inhibitor, JAK2 } \\
\text { expression inhibitor, Phospholipase inhibitor, } \\
\text { Antiseptic, Antipruritic, Antiviral, Antipyretic, } \\
\text { Antiinflammatory, Antidiabetic, Antiischemic, } \\
\text { Antipsoriatic, Antifungal, Antithyroid, Antiosteoporotic, } \\
\text { Antileprosy, Anticarcinogenic, Antialcoholic, } \\
\text { Anticataract, Antirickettsial, Hair growth stimulant, }\end{array}$ \\
\hline 13 & $\beta$-D-Glucopyranose & 13.40 & $\mathrm{C}_{6} \mathrm{H}_{12} \mathrm{O}_{6}$ & $\begin{array}{l}\text { Antidiabetic, Antioxidant, Antineoplastic, Testosterone } \\
17 \text { beta-dehydrogenase (NADP+) inhibitor, } \\
\text { Antiinflammatory, Wound healing agent, Antifungal, } \\
\text { Antiviral, Antibacterial, Antiuremic, Antipsoriatic, } \\
\text { Antimycobacterial, JAK2 expression inhibitor, } \\
\text { Dermatologic, Anticataract, Alopecia treatment, } \\
\text { Antineoplastic (liver cancer), Phobic disorders } \\
\text { treatment, Antituberculosic, Antirickettsial, Antibiotic } \\
\text { Anthracycline-like, Antibiotic Aminoglycoside-like, } \\
\text { Antibiotic Glycopeptide-like, Antibiotic Macrolide-like, } \\
\text { Lipoprotein lipase inhibitor, Phospholipase inhibitor }\end{array}$ \\
\hline 14 & Galacto-heptulose & 14.38 & $\mathrm{C}_{7} \mathrm{H}_{14} \mathrm{O}_{7}$ & $\begin{array}{l}\text { Antidiabetic symptomatic, Testosterone 17beta- } \\
\text { dehydrogenase (NADP+) inhibitor, Phobic disorders } \\
\text { treatment, Lipoprotein lipase inhibitor, Phospholipase } \\
\text { inhibitor, JAK2 expression inhibitor, Hair growth } \\
\text { stimulant, Antibiotic Anthracycline-like, } \\
\text { Aminoglycoside-like, Antiinflammatory-ophthalmic, } \\
\text { Antieczematic atopic, Antipruritic, Anticarcinogenic, } \\
\text { Antibacterial, Anticataract, Antiulcerative, Antiuremic }\end{array}$ \\
\hline 15 & $\begin{array}{l}\text { Ethyl } \alpha \text {-d- } \\
\text { glucopyranoside }\end{array}$ & 15.26 & $\mathrm{C}_{8} \mathrm{H}_{16} \mathrm{O}_{6}$ & $\begin{array}{l}\text { Membrane permeability inhibitor, Antitoxic, } \\
\text { Antineoplastic, Antiviral, Anticarcinogenic, } \\
\text { Antidiabetic, Lipoprotein lipase inhibitor, } \\
\text { Phospholipase inhibitor, Antithyroid, Lipoprotein } \\
\text { disorders treatment, Antiinfertility, Antialcoholic, } \\
\text { Antiseborrheic, Antibiotic (Glycopeptide, Macrolide- } \\
\text { like), Antimitotic (Podophyllotoxin-like), } \\
\text { Antiinflammatory-intestinal }\end{array}$ \\
\hline 16 & $\begin{array}{l}\text { 3,7,11,15- } \\
\text { Tetramethyl-2- } \\
\text { hexadecen-1-ol/ } \\
\text { Phytol }\end{array}$ & 16.13 & $\mathrm{C}_{20} \mathrm{H}_{40} \mathrm{O}$ & $\begin{array}{l}\text { Phobic disorders treatment, Testosterone 17beta- } \\
\text { dehydrogenase (NADP+) inhibitor, Antiulcerative, } \\
\text { Antiviral, Antiparasitic, Hair growth stimulant, } \\
\text { Antifungal, Membrane permeability inhibitor, } \\
\text { Antiseborrheic, Antioxidant, Dermatologic, Antiviral, } \\
\text { Antipsoriatic, Antibacterial, Antieczematic, Dementia } \\
\text { treatment, Antiinflammatory, Anticarcinogenic, } \\
\text { Anesthetic, Antituberculosic, Antimycobacterial, } \\
\text { Phospholipase inhibitor, Antineoplastic antibiotic, } \\
\text { Antibiotic Glycopeptide-like }\end{array}$ \\
\hline 17 & $\begin{array}{l}\text { 10-Methyl-E-11- } \\
\text { tridecen-1-ol } \\
\text { propionate }\end{array}$ & 16.56 & $\mathrm{C}_{17} \mathrm{H}_{32} \mathrm{O}_{2}$ & $\begin{array}{l}\text { Phobic disorders treatment, Antieczematic, Lipoprotein } \\
\text { lipase inhibitor, Antisecretoric, Testosterone 17beta- } \\
\text { dehydrogenase (NADP+) inhibitor, Antiinflammatory- } \\
\text { intestinal, Antithrombotic, Antiulcerative, Hair growth } \\
\text { stimulant, Antiviral, Antipruritic, Anesthetic, } \\
\text { Antiseborrheic, Antifungal, Dermatolog ic, } \\
\text { Antiparasitic, Antipsoriatic, Anticataract, Antiuremic, } \\
\text { Antineoplastic antibiotic, Antibiotic Glycopeptide-like, }\end{array}$ \\
\hline
\end{tabular}




\section{8}

3-O-Methyl-d-

\begin{tabular}{|l|l|} 
glucose \\
\\
\end{tabular}

18.34

$\mathrm{C}_{7} \mathrm{H}_{14} \mathrm{O}_{6}$

19

Hexanoic acid, 2-

19.16

ethyl-

\section{0}

Undecanoic acid

19.40

$\mathrm{C}_{11} \mathrm{H}_{22} \mathrm{O}_{2}$
8,11,14-

Eicosatrienoic acid, (ZZZ)-

17-Octadecynoic acid

22.38

$\mathrm{C}_{20} \mathrm{H}_{34} \mathrm{O}_{2}$

25.48

$\mathrm{C}_{18} \mathrm{H}_{32} \mathrm{O}_{2}$

\section{3}

$1,2-15,16-$

Diepoxyhexadecane
$26.97 \quad \mathrm{C}_{16} \mathrm{H}_{30} \mathrm{O}_{2}$
Lipoprotein lipase inhibitor, Phospholipase A1 inhibitor, Hair growth stimulant, Wound healing agent, Antiseptic, Diuretic inhibitor, Antipruritic (nonallergic), Antidiabetic, Antiarthritic, Antiseborrheic, Antiparasitic, Antiviral, Antibiotic Anthracycline-like, Antifungal, Aminoglycoside-like, Antineoplastic (thyroid cancer), Antidiabetic symptomatic, Antinephritic, Antibacterial, Antieczematic, Antiinflammatory- ophthalmic, Anticarcinogenic, Antirickettsial, Anticataract

Antieczematic, Lipoprotein lipase inhibitor, Hair growth stimulant, Testosterone 17beta-dehydrogenase (NADP+) inhibitor, Antiviral, Antiseborrheic, Aspergillus nuclease S1 inhibitor, Antipsoriatic, Antiinflammatory, Anesthetic, Antitoxic, Antiuretic, Alopecia treatment, Antithrombotic, Antiseptic, Antiulcerative, Antipyretic, Dermatologic, Antineurotic, Antiinflammatory, Antidiabetic, Anticataract, Antialcoholic, Cancer associated disorders treatment (Anticarcinogenic), Antifungal, Antileprosy, Antibiotic Glycopeptide-like, Carbapenem-like, 3( or 17)betahydroxysteroid dehydrogenase inhibitor,

Phobic disorders treatment, Antieczematic, Lipoprotein lipase inhibitor, Testosterone 17beta-dehydrogenase (NADP+) inhibitor, Antiseborrheic, Antiinflammatory, Anesthetic, Alopecia treatment, Hair growth stimulant, Phospholipase A2 inhibitor, Anticarcinogenic, Antituberculosic, Antiviral, Antineoplastic, Antithyroid, Antidiabetic, Antiallergic, Antieczematic, Antirickettsial, Antibacterial, Antibiotic Glycopeptide, Carbapenem, and Glycopeptide-like

Antieczematic, Lipoprotein lipase inhibitor, Phobic disorders treatment, Antiinflammatory, Antithrombotic, Antiseborrheic, Phospholipase inhibitor, Antipsoriatic, Antiviral, Antimycobacterial, Antipruritic, Antifungal, Antiuremic, Antidiabetic, Antiinflammatory, Antitoxic, Anticarcinogenic, Antiparkinsonian, Antinephritic, Anticataract, Antileprosy, Antioxidant, Antipyretic, Antirickettsial, Antibacterial, Antiuremic, Antiseptic

Lipoprotein lipase inhibitor, Prostaglandin-E2 9reductase inhibitor, Phobic disorders treatment, Antimutagenic, Antiseborrheic, Antiviral, Antisecretoric, Antithrombotic, Antiinflammatory, Antipsoriatic, Alopecia treatment, Dermatologic, Phospholipase inhibitor, JAK2 expression inhibitor, Hair growth stimulant, Antitoxic, Antiuremic, Antituberculosic, Antiinflammatory, Antipyretic, Antiseptic, Antineoplastic, Antiparasitic, Anticataract

Antieczematic, Lipoprotein lipase inhibitor, Prostaglandin-E2 9-reductase inhibitor, Phobic disorders treatment, Antimutagenic, Testosterone 17beta-dehydrogenase (NADP+) inhibitor, Antitoxic, Antiseborrheic, Membrane permeability inhibitor, Antithrombotic, JAK2 expression inhibitor, Antiviral, Phospholipase inhibitor, Antipsoriatic, Alopecia treatment, Antiinflammatory, Antiulcerative, Hair growth stimulant, Antibiotic Glycopeptide-like 


\begin{tabular}{|c|c|c|}
\hline 4 & $\begin{array}{l}\text { d-1-Xylitol,1-O- } \\
\text { undec-10-enoyl- }\end{array}$ & 28.01 \\
\hline
\end{tabular}

Oxirane[(hexadecyl $30.01 \quad \mathrm{C}_{19} \mathrm{H}_{38} \mathrm{O}_{2}$ oxy) methyl]-

26

\begin{tabular}{|l|l|l}
$\begin{array}{l}4,8,12- \\
\text { Tetradecatrien-1-01,5 } \\
\text { 9,13-trimethyl- }\end{array}$ & 31.58 & $\mathrm{C}_{17} \mathrm{H}_{30} \mathrm{O}$ \\
& &
\end{tabular}

Antitoxic, Antieczematic, Antiinfective, Lipoprotein lipase inhibitor, Antidiabetic, Antiviral, JAK2 expression inhibitor, Antineoplastic, Alopecia treatment, Anticataract, Antiseborrheic, Antifungal, Antibacterial, Wound healing agent, Antipsoriatic, Hair growth stimulant, Antipyretic, Antileukemic, Anticataract, Antineoplastic (liver cancer), Antirickettsial, Antithyroid, Antidiabetic, Antibiotic Aminoglycoside, Glycopeptide-like,

Phobic disorders treatment, Antieczematic, Antiinflammatory, Antiseborrheic, Lipoprotein lipase inhibitor, Anesthetic, Anticataract, Antiuremic, Antipruritic, Antipsoriatic, Alopecia treatment, JAK2 expression inhibitor, Wound healing agent, Hair growth stimulant, Antidiarrheal, Antiacne, Antidiabetic, Antibacterial, Antifungal, Antiallergic, Antileprosy, Antipyretic

Testosterone 17beta-dehydrogenase (NADP+) inhibitor, Phobic disorders treatment, JAK2 expression inhibitor, Antiseborrheic, Alopecia treatment, Antipsoriatic, Antieczematic, Antibacterial- (ophthalmic), Lipoprotein lipase inhibitor, Antileprosy, Phospholipase inhibitor, Antiacne, Anticarcinogenic, Anticataract, Hair growth stimulant, Antineoplastic antibiotic, Antibiotic Macrolide, Oxazolidinone, Anthracycline-like

*PASS: Prediction of Activity Spectra for Substances; **IMPPAT: Indian Medicinal Plants, Phytochemistry and Therapeutics; NCIt: NCI Thesaurus; HMDB: Human Metabolome Database; ChEBI: Chemical Entities of Biological Interest; MeSH: Medical Subject Headings.

\section{Discussion}

It is important in Ayurvedic approach to examine psychosomatic constitutions in assessing individual's health for planning preventive measures or treatment regimen including selecting diet, type of medicine, etc. Three kinds of primary body constitutions or traits (prakriti) have been defined based on three 'doshas' (humors), viz, Vata, Pitta, and Kapha. Any imbalance in these dosha results into a disease. To restore the balance, Ayurveda recommends acustomized therapy based on the 'prakriti' of that individual (19, 20).Treatment according to Ayurveda is therefore very individualized; thereby making it difficult to conduct a large population based clinical study. Since reactive oxygen species (ROS) produced in the body are composed of many species, such as, oxygen ions, peroxides, hydroxyl radicals, etc.; one would require a combination of antioxidants to quench these altogether. Plant polyphenolics though are good source of antioxidants, but have different abilities to quench all species of ROS (21-23). Therefore, one may need to use a combination of phytochemicals. It demands that one herb or one drug would not cure the imbalance of 'dosha'. Hence, traditionally, in most of the cases, a combination of herbs and plants (which are even part of staple food) are recommended for treatment (24). This is perhaps the most ancient recommendation record for a "Combinatorial and Mutlti-targted Therapy". It is quite possible that a so called crude herbal formulation has a combination of compounds, where one compound either potentiates the effect of other, or increases the bioavailibilty, or reduces the toxicity (25). In the classics, 63 major combinations of doshas have been listed and this complexity needs to be considered to elicit therapeutic effects. For example, migraine, low back pain, or arthritis of the knee can occur from vata imbalance. These diseases have different pathophysiological mechanisms, manifesting in different body regions. To treat these conditions, drugs have to act on the selected region are chosen. Compounds made from groups of plants are then combined in prescribed ways to achieve the desired effects (26). Ayurvedic medicines exist in different formats, including decoctions, powders, pastes, fermented products, tablets, and medicinal clarified butter (Ghee). The formats used, whether liquids, pastes or tablets are linked to preparations' efficacy. If the format is changed, then the desired effect may be lost and potential side effects created. In general, Dipan (digestion) and pachan (assimilation) enhancing drugs are considered good for pacifying the vitiated / imbalanced doshas (27).

A large number of medicinal plants have been checked for the phytocompounds present in the active parts or whole plant by various scientific techniques/ methods in the past fifty years. GC-MS analysis of 
phyto-extracts is a very confirmatory study of identifying phytocompounds of different molecular structures. These phytochemicals broadly belong to 6-8 types of compounds like, glycosides, fatty acids, alkaloids, etc. groups. According to Renukadevi and Sultana (28), Indigofera tinctoria leaf extract contains bioactive compounds like flavonoids, saponins, tannins, steroidal terpenes, phenols and anthroquinone. In present study, ethanolic extract of leaves of Indigofera tinctoria also showed presence of flavonoids, glycosides, saponins and terpenoids. Various extracts of leaves of Indigofera tinctoria exhibit antiulcer activity in rat (29). Renukadevi and Sultana (28) also reported the antibacterial, antioxidant and cytotoxic activity of Indigofera tinctoria leaf extract.

Present GC-MS studies of Indigofera tinctoria leaf extract revealed 26 defined chemical structures which have been recognized for specific therapeutic activities. Out of these 26 phytocompounds, a maximum of 4 numbers fatty acid group followed by 2 numbers belonging to glycoside group/derivatives and so on. Similar types of compounds were reported from Evolvulus alsinoides L. (30, 31). Evolvulus species were reported for treatment of coughs and colds, wound healing, burns, cuts, scorpion bites, illnesses associated with the nervous system, including epilepsy, memory loss, mental disorders, anthelmintic, anti-inflammatory and antioxidant activities $(32,33)$. The present study with Indigofera, reported maximum phytocompounds that are efficiently used as a part of treatments to promote therapeutic activities like wound healing, antidiabetic, antiallergic, antifungal, antibacterial and anticataract, etc. The phytochemicals relating to hair disorder treatments, such as testosterone 17betadehydrogenase (NADP+) inhibitor, antiseborrheic, antiandrogenic, antidermatological and JAK2 expression inhibitor activities are significantly important for the use of the plant. Similarly, therapeutic activity revealed from some phytocompounds as an antibiotic (Glycopeptide, Carbapenem Macrolide-like), Antimitotic (Podophyllotoxin-like), Antineoplastic antibiotic, etc. also highlight the multifunction nature of the plant phytoconstituents. As per literature, Glycopeptide is used if infection caused by methillinresistant Staphylococcus aureus, and Carbapenem is active against many aerobic and anaerobic gram positive and negative microorgamisms (34). Thus, this study opens opportunity for learning a comparison between select phyto-compounds and reference structures (antibiotics) with class of similarity and that will help to reduce dependency on antibiotics. According to pubchem reports, 1,2,3 Butanetriol is used as general adhesive, soap, binding, rinsing and softening reagent. NCI Thesaurus reports 3,6Octadecadiynoic acid, methyl ester as androstenediol, which is direct metabolite of dehydroepiandrosterone (DHEA) and a precursor for testosterone with immunostimulatory activity and negligible androgenic activity.

The potential of PASS in predicting a biological activity spectrum (BAS) of natural product has not been fully explored and in that, to correlate the BAS of the major phytocompounds is respectively low (35). So the present attempt in representing the identified phytocompounds of Indigofera tinctoria leaf extract and the predicted biological /therapeutic activities of these compounds will enable newer drug formulation and disease management with further in-silico and clinical studies.

\section{Conclusion}

In the present study, twenty six phytocompounds have been recognized from ethanolic extract of Indigofera tinctoria L. leaves by GC-MS analysis mostly confirming to the classically confirmed phytochemical groups. Based on the results and discussion in this research paper, it can be concluded that Indigofera tinctoria bioactive phytocompounds have high probable therapeutic value to cure various ailments. However, further studies are needed to study similarity class of phytocompounds associated with use of antibiotics and relevant compounds to find the efficacy of plant drug in more pharma products.

\section{Acknowledgement}

The authors express sincere thanks to the SAIF laboratories, IIT, Bombay for the help received while carrying out the GC-MS study. Thanks to authorities of COCSIT, Latur for the moral support and inspiration during research work.

\section{References}

1. Warrier P.K, Nambiar V.P.K, Ramankatty C. Indian Medicinal Plants. Orient Longman Private Limlited, Chenni. 2007; 210-213

2. Tamilselvi N, Krishnamoorthy P, Dhamotharan R, Arumugam P, Sagadevan, Analysis of total phenols, total tannins and screening of phytocomponents in Indigofera aspalathoides (Shivanar Vembu) Vahl EX DC. Journal of Chemical and Pharmaceutical Research. January, 2012; 4(6); 3259-3262

3. Motamarri S.N, Karthikeyan M, Rajasekar S, Gopal $\mathrm{V}$, Indigofera tinctoria Linn - A Phytopharmacological Review. International Journal of Research in Pharmaceutical and Biomedical Sciences. January, 2012; 3(1); 164-169

4. Gerometta E, Grondin I, Jicqueline S, Frederich M, Gauvin-Bialecki A,A review of traditional uses, phytochemistry and pharmacology of the genus Indigofera. Journal of Ethanopharmacology. January, 2020; 253; 1-77

5. Dembitsky V.M, Glorivozova T.A, Poroikov V.V, Novel Antitumor Agents: Marine Sponge Alkaloids, Their Synthetic Analogs and Derivatives. Mini Rev Med Chem. March, 2005; 5(3); 319-36 
6. Zotchev S.B, Brautaset T, Sletta H, Nedal A, Borgos S.E.F, Degnes K.F, Bakke I, Improved Antifungal Polyene Macrolides via Engineering of the Nystatin Biosynthetic Genes in Streptomyces noursei. Chemistry \& Biology. November, 2008; 15(11); 1198-1206

7. William Evans. Trease and Evans Pharmacognosy. 16th edition. London; Saunders Publishers. 2009;194-332

8. Kramida A, Ralchenko Yu, Reader J, NIST ASD Team. NIST Atomic Spectra Database (version 5.7.1), dated 18-12-2019 time 12:15 IST

9. Kim S, Thiessen P.A, Bolton E.E, Chen J, Fu G, Gindulyte A, Bryant S.H, PubChem substance and compound databases. Nucleic Acids Research. January, 2016; 44(D1); D1202-13

10. Hähnke V.D, Kim S, Bolton E.E, PubChem chemical structure standardization. Journal of Cheminformatics. August, 2018; 10(1); 36

11. Lagunin A, Stepanchikova A, Filimonov D. and Poroikov V, PASS: Prediction of activity spectra for biologically active substances. Bioinformatics. August, 2000; 16(8); 747-48

12. Mohanraj K, Karthikeyan B.S, Vivek-Ananth R.P, Chand R.P.B, Aparna S.R, Mangalapandi P, Samal A, IMPPAT: A curated database of Indian Medicinal Plants, Phytochemistry and Therapeutics. Scientific Reports. March, 2018; 8(1); 4329

13. NCI Thesaurus (NCIt) provides reference terminology https://ncit.nci.nih.gov/ncitbrowser/ $\mathrm{C} \quad \mathrm{o} \quad \mathrm{n}$ c e $\mathrm{p}$ t $\mathrm{R}$ e $\mathrm{p}$ o $\mathrm{r}$ t. dictionary=NCI_Thesaurus\&ns=NCI_Thesaurus\&c ode $=$ C561, dated 18-05-2020 time 13:05 IST

14. The Human Metabolome Database (HMDB) electronic database http://www.hmdb.ca/ metabolites/HMDB0000562, dated 02-05-2020 time 17:45 IST

15. Chemical Entities of Biological Interest (ChEBI): database and ontology of molecular entities http:// ww w.ebi .ac.uk/chebi/s e a r ch Id.do? chebiId=CHEBI:16737, dated 02-05-2020 time 18:45 IST

16. Medical Subject Headings (MeSH) https:// www.ncbi.nlm.nih.gov/mesh/68019325, dated 12-05-2020 time 14:15 IST

17. A unique bioinformatics and cheminformatics resource. http://www.drugbank.ca/drugs/DB01524, dated 02-05-2020 time 15:05 IST

18. Wale N, Ning X, Karypis G. Trends in chemical graph data mining. Managing and mining graph data. 2010; 581-606.

19. Lad V.D. Textbook of Ayurveda. 1st edition. The Ayurvedic Institute, Albuquerque, New Mexico, $2002 ; 13 p$

20. Garodia P, Ichikawa H, Malani N, Sethi G, Aggarwal B.B, From ancient medicine to modern medicine: ayurvedic concepts of health and their role in inflammation and cancer. J Soc Integr Oncol. January, 2007; 5(1); 25-37

21. Kondratyuk T.P, Pezzuto J.M, Natural Product Polyphenols of Relevance to Human Health. Pharm Biol. January, 2004; 42(1); 46-63

22. Spencer J.P, Abd El M.M, Minihane A.M, Mathers J.C, Biomarkers of the intake of dietary polyphenols: strengths, limitations and application in nutrition research. Br J Nutr. January, 2008; 99(1); 12-22

23. Pande K.B, Rizvi S.I, Plant polyphenols as dietary antioxidants in human health and disease. Oxid Med Cell Longev. November-December, 2009; 2(5); 270-278

24. Dhruva A, Frederick M.H, Christine M, Ted J.K, Bodeker G, Donald A, Lad V, Shelley R.A, Correlating Traditional Ayurvedic and Modern Medical Perspectives on Cancer: Results of a Qualitative Study. The Journal of Alternative and Complementary Medicine. May, 2014; 20(5); 364-370

25. Grazia G, Penna I, Perotti V, Anichini A, Tassi E, Towards combinatorial targeted therapy in melanoma: from pre-clinical evidence to clinical application (Review). Journal of oncology. September, 2014; 45(3); 929-949

26. Kumar S, Dobos G.J, Rampp T, The significance of ayurvedic medicinal plants. Journal of EvidenceBased Complementary \& Alternative Medicine, July, 2016; 22(3); 495-501

27. Singh A.K, Gupta A.K, Singh P.K, Manish A, Comprehensive study of basic principles of Ayurveda. International Journal of Applied Ayurved Research. March, 2014; 1(3);1-20

28. Renukadevi K.P, Sulthana S.S, Determination of Antibacterial, Antioxidant and Cytotoxicity Effect of Indigofera tinctoria on Lung Cancer Cell Line NCI-h69. International Journal of Pharmacology, March, 2011; 7(3); 356-362

29. Umarani N, Ilango K, Valentina P, Sunitha P.G, Anandarajagopala K, Antiulcer activity of various extracts of leaves of Indigofera tinctoria. Int. J. Chem. Sci. January, 2008; 6(1); 281-284

30. Elangovan K, Supriya K, Murugesan K, Aravind R, Screening of phytochemicals and in vitro antioxidant activity of Evolvulus alsinoides L. J Acad Industr Res. September, 2013; 2(4); 230-234

31. Gomathi D, Kalaiselvi M, Ganesan R, Kanakasabapathi D, Chandrasekar U, GC-MS analysis of bioactive compounds from the whole plant ethanolic extract of Evolvulus alsinoides (L.). J Food Sci Technol. February, 2015; 52(2); 1212-1217

32. Ramya S, Alaguchamy N, Maruthappan V.M, Sivaperumal R, Sivalingam M, Krishnan A, et al,. Wound healing ethnomedicinal plants popular 
among the malayali tribes in Vattal Hills, Dharmapuri, TN, India. Ethnobotanical Leaflets. October, 2009; 13(1); 1257-1271

33. Dhanalekshmi U.M, Kishore G.P, Raja M.D, Reddy P.N, Evaluation of wound healing potential and antimicrobial activity of ethanolic extract of Evolvulus alsinoides. Ann Biol Res. March, 2010; $1(2) ; 49-61$
34. Itzhak B, Wexler H.M, Goldstein J.C.E, Antianaerobic Antimicrobials: Spectrum and Susceptibility Testing. Clin Microbiol Rev. July, 2013; 26(3); 526-546

35. Pramely R, Raj T.L.S, Prediction of biological activity spectra of a few phytoconstituents of Azadirachta indicia A. Juss. J Biochem Tech. October, 2012; 3(4); 375-379 